\title{
Determination of the granulometric composition parameters of the grinding product in a ball mill
}

\author{
Rashid Sharapov ${ }^{*}$ \\ Moscow State University of Civil Engineering, Yaroslavskoe shosse, 26, Moscow, 129337, Russia
}

\begin{abstract}
The article deals with the issue of the formation of the grain composition of the comminuted material inside the drum of a ball mill. The approach to the calculation of the grain composition at its transition from a larger fraction from a smaller fraction is presented. The proposed approach was tested on an industrial cement ball mill and confirmed on an experimental installation. This approach allows you to determine the parameters of the kinetics of grinding, depending on the design parameters used ball mills.
\end{abstract}

\section{Introduction}

The production of the fine powders is a very important task for any sphere of modern industry. One of the most technologically advanced powder, that produce humanity for its use is Portland cement [1-5]. The production of cement is accompanied by complex processes of transformation of raw materials into finish product. The material passes the stage of grinding, homogenizing, calcining and grinding again the almost finished product in a finely dispersed powders.

A large number of devices proposed by various researchers for grinding the components of the cement and the Portland cement clinker and additives [6-11]. At the same time the requirements for quality of the final product to grinding equipment are very high level. To fulfil these requirements all different types of the grinding equipment works in a closed cycle grinding. Therefore, as practice has shown the most important element of technological systems of grinding are closed circuit ball mills. These type of ball mills in the coming decades will remain the basic units for grinding cement clinker and additives [6].

\section{Teoretical}

The main task of modeling the grinding process is predicting the granulometric composition of the finished product. This product depends on the granulometric composition of the source material, the constructive and technological parameters of the ball mill and separator. Predicting the granulometric composition of the finished product

\footnotetext{
* Corresponding author: ptdm_zavkaf@mail.ru
} 
will allow to choose the optimal configuration of necessary equipment and correct operation modes.

The most complete and informative characterization of the grinding process in a ball mill is a matrix of the grinding process $P=\left(p_{i j}\right)$. Each element of this matrix $p_{i j}$ equal to the relative share of the $j$-th fraction of bulk raw materials passing into the $i$-th fraction of the grinding product $(i, j=1,2, \ldots, m$, where $m$ corresponds to the smallest fraction of the grinding material). All the elements of the matrix of grinding, for which $i>j$ is equal zero, as the particles in the grinding process of their factions to a larger faction can not pass. It can be explain because the reverse process in the mill does not occur. As a result, the grinding matrix has a lower triangular form [12-16]:

$$
P=\left[\begin{array}{cccc}
p_{11} & 0 & 0 & 0 \\
p_{21} & p_{22} & 0 & 0 \\
p_{31} & p_{32} & p_{33} & 0 \\
- & - & - & - \\
p_{m 1} & p_{m 2} & & p_{m m}
\end{array}\right] .
$$

Standing on the main diagonal of the matrix elements $p_{j j}$ show the relative (by mass) of the particles of the $j$-th fraction, remaining in it because of weak grinding the particles. The elements of the matrix $R$ satisfy the conditions of normalization, reflecting the law of conservation of mass of individual fractions: total probability of transition of particles of the $j$-th fraction into smaller fractions $i \geq j$ is equal to unit:

$$
\begin{gathered}
p_{11}+p_{21}+\ldots+p_{\mathrm{m} 1}=1 \\
p_{22}+p_{32}+\ldots+p_{\mathrm{m} 2}=1 \\
p_{m m}=1 .
\end{gathered}
$$

The transformation of the granulometric composition of the material as a result of grinding process in a ball mill is described by the matrix equation:

$$
f^{\prime}=P f
$$

where $f$ and $f$ - are the vectors of the granulometric composition of the disperse material at the inlet to the ball mill and out of it:

$$
f=\left[\begin{array}{c}
f_{1} \\
f_{2} \\
\cdots \\
f_{m}
\end{array}\right], \quad f^{\prime}=\left[\begin{array}{c}
f_{1}^{\prime} \\
f_{2}^{\prime} \\
\cdots \\
f_{m}^{\prime}
\end{array}\right] .
$$

Here $f_{1}, f_{2}, \ldots, f_{m}$ and $f_{1}{ }^{\prime}, f_{2}{ }^{\prime}, \ldots, f_{m}{ }^{\prime}$ are relative the mass share of the particles of material falling into respective fractions. In formulas (1)...(4) adopted the reverse order numbering of the fractions, i.e. in descending order of particle size.

Because of the extreme complexity and variety of processes occurring in ball mills, the theoretical definition of the matrix of grinding currently is not possible. In connection with this very urgent task of reconstruction of the matrix of grinding $P$ on the experimental data obtained on a real ball mill working production.

The matrix equation (3) is equivalent to the system of $m$ linear equations: 


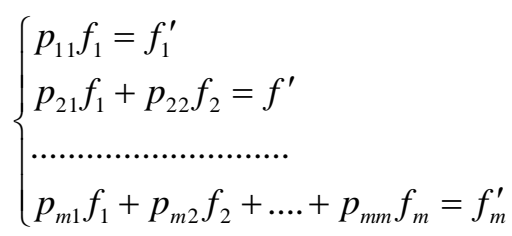

of which independent are only $m-1$ equations, because the addition of linear equations (5) subject to conditions (2) gives the identity:

$$
f_{1}+f_{2}+\ldots+f_{m}=f_{1}{ }^{\prime}+f_{2}{ }^{\prime}+\ldots+f_{m}{ }^{\prime}=1 .
$$

Thus, at a certain granulometric composition $f$ and $f^{\prime}$ to determine $m(m+1) / 2$ elements of the matrix of grinding in ball mill $P$ we have $2 m-1$ equations. It follows that the unique reconstruction of the elements of the matrix of grinding on particle size distribution at the entrance to the mill and exiting is possible only by dividing the material into two fractions large and small. In this case

$$
P=\left[\begin{array}{cc}
p_{11} & 0 \\
p_{21} & p_{22}
\end{array}\right], \quad p_{11}=\frac{f_{1}^{\prime}}{f_{1}}, p_{21}=1-p_{11}, \quad p_{22}=1 .
$$

When $m>2$ the knowledge of only the input or output particle size distribution, the mill charge inside the mill drum material is not enough.

In the article [10] suggested a method of reconstruction of the matrix of the grinding on the results of the $m$-fold transmission of material through the mill drum. However, the thus obtained experimental data in determining the elements of the initial row of matrix grinding are used only partially, which reduces the reliability of the results. It should also be noted the inability of the proposed experiment in terms of current production. This can be explained by the fact that in the actual production, it is impossible to change either the design features of the equipment or the technological process or the properties of comminuted material.

On the basis of experimental data obtained on industrial installations, using matrix method, we define the kinetics of the grinding process in closed circuit ball mills. For this purpose each chamber of the ball mill are presented in the form of $n$ equal-length parallel working sections, the length of which corresponds to the step of removing the chart of grinding. Extended milling diagram provided in the form of a matrix of size $m \times n$ :

$$
F=\left(f_{j k}\right)=\left[\begin{array}{llll}
f_{11} & f_{12} & \ldots & f_{1 n} \\
f_{21} & f_{22} & \ldots & f_{2 n} \\
\ldots & \ldots & \ldots & \ldots \\
f_{m 1} & f_{m 2} & \ldots & f_{m n}
\end{array}\right] .
$$

Here the index $j=1,2 \ldots, m$ corresponds to the number fraction of the grinding material, and the index $k=0, \ldots, n-1$ corresponds to the cross-sections along the length of the mill drum, in which selected material samples for making matrix of the grinding. The section with index $k$ is also an input section $(k+1)$-th section of the mill.

Thinking that the matrix of the grinding in separate sections $P_{\mathrm{c} k}$ does not depend of the fractional composition of the material and therefore have the same form $P_{c k}=P_{c}$, we write the equation of the transformation of the granulometric composition of material:

$$
f_{k+1}=P_{c} f_{k}, k=0,1,2, \ldots, n-1 .
$$


The resulting matrix of grinding for the whole chamber of the mill is determined by the following ratio:

$$
P=P_{c 1} \cdot P_{c 2} \cdots P_{c k}=P_{c}^{n},
$$

where $P_{c}$ - matrix of grinding for each step of sampling in the ball mill.

Matrix of grinding considering the fact that in modern ball mills consist of 2 cameras and for the whole the mill matrix of grinding is:

$$
P=P_{1} P_{2},
$$

where $P_{1}=P_{c 1}^{n 1}, P_{2}=P_{c 2}^{n 2}-$ matrix of grinding along the length of the chambers of ball mill, respectively, the first and second chambers.

Matrix equations (8) are equivalent to $n$ systems of linear equations:

$$
\begin{aligned}
& p_{11} f_{1 k}=f_{1 k+1} \\
& p_{21} f_{1 k}+p_{22} f_{2 k}+f_{2 k+1} \\
& p_{31} f_{1 k}+p_{32} f_{2 k}+p_{33} f_{3 k}=f_{3 . k+1} \\
& \ldots \ldots \ldots \ldots \ldots \ldots \ldots \ldots \ldots \ldots \ldots \ldots \ldots \ldots \ldots \ldots \ldots \ldots \ldots \ldots \ldots \ldots \ldots \ldots \ldots \ldots \ldots \ldots \\
& p_{m 1} f_{1 k}+p_{m 2} f_{2 k}+p_{m 3} f_{3 k}+\ldots+p_{m m} f_{m k}=f_{m k+1} \\
& k=0.1, \ldots, n-1,
\end{aligned}
$$

which must be supplemented by the normalization conditions (2). The total number of independent equations $n(m-1)+m$ greatly exceeds the number to be determined of the elements of the matrix of grinding $P_{c}$. These values are obtained by researchers when removing the charts grinding in both chambers of the ball mill. The accuracy is higher, the more often we take the chart of grinding in the drum ball mill. However, taking into account the inevitability of random errors when removing the diagram of grinding (such as the enrichment of sieving large fraction of the products of burnout, large particles in interlining space made bring from outside researchers, etc.), we must do all of the above a specific set of equations (10) using the least squares method [15, 17-21]. Summing the squares of the residuals, i.e. the difference of the left and right sides of each of equations of system (10) for all partitions, we get:

$$
\begin{aligned}
& S_{1}=\sum_{k=0}^{n-1}\left(p_{11} f_{1 k}-f_{1 k+1}\right)^{2}, \\
& S_{2}=\sum_{k=0}^{n-1}\left(p_{21} f_{1 k}+p_{22} f_{2 k}-f_{2 k+1}\right)^{2}, \\
& S_{m-1}=\sum_{k=0}^{n-1}\left(p_{m-11} f_{1 k}+p_{m-12} f_{2 k}+\ldots+p_{m-1 m-1} f_{m-1 k}-f_{m-1 k+1}\right)^{2} .
\end{aligned}
$$

Differentiating the functions $S_{1}, S_{2}, \ldots, S_{m-1}$ within them the elements of the matrix of grinding, obtain the system of normal equations to determine these equations.

In particular, the formula for calculating $p_{11}$ has the form:

$$
p_{11}=\sum_{k=0}^{n-1}\left(f_{1 k+1} f_{1 k}\right) / \sum_{k=0}^{n-1} f_{1 k}^{2} .
$$


The system of equations for the determination of $p_{21}$ and $p_{22}$ can be written thus:

$$
\begin{aligned}
& \left(\sum f_{1 k}^{2}\right) p_{21}+\left(\sum f_{2 k}\right) p_{22}=\sum f_{2 k+1} f_{1 k} \\
& \left(\sum f_{1 k} f_{2 k}\right) p_{21}+\left(\sum f_{2 k}^{2}\right) p_{22}=\sum f_{2 k+1} f_{2 k}
\end{aligned}
$$

The elements of the last row of the matrix of grinding is calculated using the found least-squares method, the elements of the other rows by using the normalization conditions (2).

\section{Conclusion}

Using found, as described above, the matrix for grinding for the sections of the chambers of ball mill it is possible to investigate the kinetics of the grinding process of the material along each of them, respectively, along the mill as a whole. This will allow us to choose the appropriate size and composition of the grinding media in the chambers of the mills, lining each chamber of the mill, the suction conditions inside the mill drum, to optimize the size of the incoming material and so on.

This work was financially supported by Ministry of Science and Higher Education of the Russian Federation (\#NSh-3492.2018.8).

\section{References}

1. R.R. Sharapov, P.D. Kapyrin S.Y. Lozovaya, V.V. Yadykina, A.M. Agarkov, MATEC Web of Conferences 5. "5th International Scientific Conference "Integration, Partnership and Innovation in Construction Science and Education", IPICSE 2016". 03007. (2016)

2. A.A. Romanovich, Wor. Ap. Sc. J. 25. 2. (2013)

3. E. Hanke, Wor. Cem. 17. 3. (1986)

4. W. H. Duda, Cement-data-book. Band 1 (Internationale Verfahrenstechniken der Zementindustrie, Wiesbaden, Berlin: Bauverlag) (1985)

5. Global Projects. Wor. Cem. December. (2007)

6. Subeggve, ZKG Int. 2. (2004)

7. V.I. Telichenko, R.R. Sharapov, S.Y. Lozovaya, V.I. Skel, MATEC Web of Conferences 5. "5th International Scientific Conference "Integration, Partnership and Innovation in Construction Science and Education", IPICSE 2016". 04040. (2016)

8. V.S. Sevost'yanov, A.A. Goncharov, V.P. Potelezhko, Gl \&Cer. 50, 2. (1993)

9. V.A. Uvarov, G1 \& Cer. 63. 7-8. (2006)

10. Grinding Mill - Rod, Min. mag. 147. 9. (1982)

11. A. Nerholm, Symposium on cement manufacture. Moscow: Niicement Research Institute, 1979

12. V. Mizonov, V. Zhukov, S. Bernotat, Simulation of Grinding: new approaches. (Ivanovo: ISPEU Press, 1997)

A. Jankovic, W. Valery, E. Davis, Min. Eng. 17 (2004)

13. L.V. Ryadinskaya, S.Y. Lozovaya, V.S. Sablin, Int. J. App. Eng. Res. 9. 22. (2014) 
14. N.N. Kalitkin, Numerical methods. (Moscow: Nauka, 1998)

15. V.S. Bogdanov, Y.M. Fadin, S.Y. Lozovaya, S.S. Latyshev, N.E. Bogdanov, O.S. Vasilenko,. Int. J. Phar. \& Tech. 8. $\underline{3}$. (2016)

16. ACM. 6. (1999)

17. R.R. Sharapov, I.V. Shrubchenko, A.M. Agarkov, Int. J. Ap. Eng. Res. 10. 2. (2015)

18. H.J. Nielsen, S. Tsuchiya, ZKG Int. 54. 1. (2001)

19. R. McDowell, I. Mensz, P. \& Q. 12. (1987)

20. E. Onuma, T. Furukawa, Wor. Cem. 10. (1982) 